(1)

CrossMark

\title{
Breath biomarkers in asthma: we're getting answers, but what are the important questions?
}

\author{
Stephen J. Fowler $\mathbb{B}^{1}$ and Peter J. Sterk ${ }^{2}$ \\ Affiliations: ${ }^{1}$ Division of Infection, Immunity and Respiratory Medicine, School of Biological Sciences, Faculty \\ of Biology, Medicine and Health, University of Manchester, and Manchester Academic Health Science Centre \\ and NIHR Biomedical Research Centre, Manchester University Hospitals NHS Foundation Trust, Manchester, \\ UK. ${ }^{2}$ Dept of Respiratory Medicine, Amsterdam UMC, University of Amsterdam, Amsterdam, The Netherlands.
}

Correspondence: Stephen J. Fowler, Education and Research Centre, Wythenshawe Hospital, Manchester M23 9LT, UK. E-mail: Stephen.fowler@manchester.ac.uk

@ERSpublications

Reports of potential biomarkers in breath are rapidly accumulating, such that systematic reviews are now feasible. We should now take stock and ensure every future study design is able to contribute a step forward towards future clinical application. http://bit.ly/2khqrWi

Cite this article as: Fowler SJ, Sterk PJ. Breath biomarkers in asthma: we're getting answers, but what are the important questions? Eur Respir J 2019; 54: 1901411 [https://doi.org/10.1183/13993003.01411-2019].

Over recent years, the field of breathomics has matured such that systematic reviews are not only viable, but considered important enough for dissemination via high impact clinical research journals such as the European Respiratory Journal. Recently, we have seen accelerating interest in this exciting biomarker discovery platform with reviews covering topics such as paediatric asthma [1], lung infection [2, 3] and fibrotic lung diseases $[4,5]$. For rapidly developing fields such as this, synoptic work provides important functions both for newcomers, by introducing and stimulating interest, but equally for established researcher teams, by highlighting both consistency and variability in findings across studies. This helps create a platform for improved, and perhaps standardised, developments in study design, sample collection and analysis, and data analysis and reporting. Invariably these reviews have highlighted the paucity of longitudinal, well controlled studies, and significant variability in sampling and analytical techniques [6].

In this issue of the European Respiratory Journal, AzIm et al. [7] report a new systematic review covering breathomics in adult asthma. They found that reports of high quality remain relatively few, with only 22 studies meeting the fairly broad inclusion criteria. Most of the included studies investigated breath for disease identification, rather than more refined targets, such as asthma phenotyping or identification of treatment responses or exacerbations. The varied methodologies employed make direct comparisons difficult and meta-analysis impossible, and perhaps because of this the authors did not attempt to list the identified volatile organic compounds (VOCs) from each study. Such summative lists would nevertheless be useful both in highlighting inconsistency and also (more encouragingly) where the same or similar discriminatory volatiles have been identified in studies using different populations, sampling and analytical techniques. One example is the different breathing techniques employed; we know for exhaled nitric oxide different expiratory flows enable targeting of different airway/alveolar compartments, and result in very different values [6]; this is very likely to be the case for breath volatiles too.

Whilst we are still in this discovery stage it is time to refine our study designs so that we can make progress towards tailored clinical application. The most commonly stated aim in current studies is to 
investigate potential breath biomarkers that can be used in the diagnosis of asthma. However, they typically report differences in breath profiles between participants with a diagnosis of asthma compared to a group without. The usual "gold standard" cited in these studies is often a "doctor's diagnosis" of asthma, which we know to be wrong in up to a third of cases [8]. Hence, this "gold standard" may be the weakest part in the chain when validating new biological diagnostic markers. This problem is found in other diseases, such as acute respiratory distress syndrome, and is a key problem in biomarker research [9].

Hypotheses addressing changes in volatiles in disease are typically constructed on expected differences due to inflammation, treatment intervention and/or even the microbiome. In this regard there are likely to be many critical differences in clinical characteristics between the asthma patients included in such studies compared to those in whom we need a diagnosis, i.e. someone presenting to their primary care physician with a wheeze and/or cough. In such studies the average age is usually well into adulthood, participants have had "established disease" perhaps for 10 years or more, and are most likely taking anti-inflammatory and bronchodilator therapy. It is therefore perhaps not surprising that so few findings have been externally validated or replicated. Nitric oxide may well have not been confirmed as a potential diagnostic in asthma if studies had only investigated its use in steroid-treated patients [10].

In fact it is debateable whether the concept of a "single diagnostic" for asthma is even credible, especially one based on inflammation when the objective diagnosis is in fact based on demonstration of variable airflow obstruction. Is it biologically plausible that a biochemical marker in the breath might tell us that there is variable airflow obstruction? Perhaps we should be ignoring the somewhat artificial concepts of asthma (or COPD), and instead target the inflammatory phenotype directly; a tactic described with successful results in the European Respiratory Journal last year by DE VRIES et al. [11]. If we do persevere with pursuing the Holy Grail of instant diagnosis, we must consider at the design stage whether we are aiming for confirmation or exclusion of disease. This differs between diseases: for lung cancer, biomarkers should primarily exclude the disease, meaning one should aim for $100 \%$ sensitivity; for asthma diagnosis, perhaps one could aim for being more specific by optimising positive predictive value.

There are many other urgent questions in asthma beyond diagnosis [12]. How do we select, titrate and monitor treatment? Who is at risk of exacerbation or severe disease? These questions are likely linked to distinct mechanistic pathways [13], which therefore might be reflected in biochemical markers. However, specifically designed, prospective studies are needed to address them, and to date few have been performed. As for diagnosis, these studies need to be hosted in the environment in which they will be performed, and compared to a clinically and biologically meaningful "gold standard".

Where can breath outperform currently available biomarkers? One area perhaps is in predicting response to monoclonal antibodies in severe asthma, such as anti-IL-5. Selection for the latter is currently based on blood eosinophils, but we know that around a fifth of those without blood "eosinophilia" still have evidence of eosinophilic airway inflammation [14, 15], and are thus potentially missing out on these treatments. On the other hand, roughly the same proportion of those meeting the prescribing criteria may not have any clinically meaningful benefit. A non-invasive and predictive marker of airway inflammation and treatment response would certainly be of use here, and preliminary data suggest that it may be achievable with breath [16].

Alternatively, the "bottom up" approach aims to discover selective volatiles or particular combinations of those in cell or animal models and then look for them in well-designed human studies. This is most obviously applicable in bacterial infection $[17,18]$ or in identifying dominant inflammatory cell signatures in asthma [19], but has not yet been translated all the way from cell to breath. Since these metabolites are likely to be (far) downstream of cellular processes, perhaps the way to go is to link exhaled markers and their patterns to transcriptomic and proteomic data that more directly represent cell biology. Such indirect anchoring of breath profiles has recently been done in relation to sputum transcriptomics [20].

As reported by AzIM et al. [7], sufficiently detailed description of technical methods, especially analytical parameters and data pre-processing, are rarely given to allow comparison between studies. Given the sensitivity of the instruments used, and variability between them, it is not surprising that few studies have validated others' findings. The only cases of external validation have occurred where the same analytical team have been used for the training and validation samples [11, 21, 22]. The recent work by SCHLEICH et al. [22] in fact is the first that has provided true external validation by gas chromatography mass spectrometry (GCMS) in a large number of patients. This was likely due not only to the tight control of analytical aspects (both chemical and statistical) but also the selection of an airway-relevant and testable hypothesis (airway inflammatory profiles). Another serious consideration for any biomarker discovery study, especially in asthma, and very recently confirmed to be important in breath, is the influence of circadian rhythm [23]. 
AzIm et al. [7] do not provide an analysis of specific VOCs that have been found to be discriminatory between disease characteristics of interest. This was beyond the scope of their already extensive work, but of interest to both clinicians and researchers. It is also of vital importance if findings are to be validated. Furthermore, when VOCs are reported some indication of the confidence in findings must be given (e.g. following Metabolomics Standards Initiative recommendations [24]). As noted above, GCMS studies have had difficulty in replicating VOCs in association with asthma. GCMS can be also used to generate fingerprints of VOCs similar to eNoses; arguably this approach may reflect complex biology better than identification of each individual metabolite.

Breathomics is perhaps at "the end of the beginning" for asthma at least; it has a "sexy" name, some promising and consistent findings, and the key questions are at last being better recognised. It is vital that studies now move forward with designs that address the needful questions in the spirit of personalised medicine and report methods that can be independently replicated across populations and laboratories. We hope that this is the beginning of a new era, and that future systematic reviews may be able to access data of sufficient quality on which to perform meta-analysis on individual or combinations of VOCs, and get us closer to clinical implementation.

Conflict of interest: S.J Fowler reports personal fees for lectures and non-financial support for meeting attendance from AstraZeneca, grants and personal fees for lectures from Boehringer Ingelheim, personal fees for lectures from Novartis and Teva, personal fees for advisory board work from Chiesi, outside the submitted work. P.J. Sterk reports being Scientific Advisor and having a formally inconsiderable interest in the start-up company Breathomix, a start-up producing eNoses and licensing a cloud application for data analysis, during the conduct of the study.

\section{References}

1 Neerincx AH, Vijverberg SJH, Bos LDJ, et al. Breathomics from exhaled volatile organic compounds in pediatric asthma. Pediatr Pulmonol 2017; 52: 1616-1627.

2 Bos LD, Sterk PJ, Schultz MJ. Volatile metabolites of pathogens: a systematic review. PLoS Pathog 2013; 9: e1003311.

3 Ahmed WM, Lawal O, Nijsen TM, et al. Exhaled volatile organic compounds of infection: a systematic review. ACS Infect Dis 2017; 3: 695-710.

4 Hayton C, Terrington D, Wilson AM, et al. Breath biomarkers in idiopathic pulmonary fibrosis: a systematic review. Respir Res 2019; 20: 7.

5 Terrington DL, Hayton C, Peel A, et al. The role of measuring exhaled breath biomarkers in sarcoidosis: a systematic review. J Breath Res 2019; 13: 036015.

6 Horvath I, Barnes PJ, Loukides S, et al. A European Respiratory Society technical standard: exhaled biomarkers in lung disease. Eur Respir J 2017; 49: 1600965.

7 Azim A, Barber C, Dennison P, et al. Exhaled volatile organic compounds in adult asthma: a systematic review. Eur Respir J 2019; 54: 1900056.

8 Aaron SD, Vandemheen KL, FitzGerald JM, et al. Reevaluation of diagnosis in adults with physician-diagnosed asthma. JAMA 2017; 317: 269-279.

9 Bos LDJ. Diagnosis of acute respiratory distress syndrome by exhaled breath analysis. Ann Transl Med 2018; 6: 33.

10 Kharitonov SA, Yates D, Robbins RA, et al. Increased nitric oxide in exhaled air of asthmatic patients. Lancet 1994; 343: 133-135.

11 de Vries R, Dagelet YWF, Spoor P, et al. Clinical and inflammatory phenotyping by breathomics in chronic airway diseases irrespective of the diagnostic label. Eur Respir J 2018; 51: 1701817.

12 Bos LD, Sterk PJ, Fowler SJ. Breathomics in the setting of asthma and chronic obstructive pulmonary disease. Allergy Clin Immunol 2016; 138: 970-976.

13 Pavlidis S, Takahashi K, Ng Kee Kwong F, et al. "T2-high" in severe asthma related to blood eosinophil, exhaled nitric oxide and serum periostin. Eur Respir J 2019; 53: 1800938.

14 George KE, Ryan DM, Keevil B, et al. A pilot study to investigate the use of serum inhaled corticosteroid concentration as a potential marker of treatment adherence in severe asthma. J Allergy Clin Immunol 2017; 139: 1037-1039.

15 Hastie AT, Moore WC, Li H, et al. Biomarker surrogates do not accurately predict sputum eosinophil and neutrophil percentages in asthmatic subjects. J Allergy Clin Immunol 2013; 132: 72-80.

16 van Bragt JJMH, de Vries R, Sterk PJ, et al. Exhaled breath for prediction of responders to mepolizumab in patients with severe asthma. Am J Respir Crit Care Med 2019; 199: A2673.

17 Lawal O, Muhamadali $\mathrm{H}$, Ahmed W, et al. Headspace volatile organic compounds from bacteria implicated in ventilator-associated pneumonia analysed by TD-GC/MS. J Breath Res 2018; 12: 026002.

18 van Oort PM, Brinkman P, Slingers G, et al. Exhaled breath metabolomics reveals a pathogen-specific response in a rat pneumonia model for two human pathogenic bacteria: a proof-of-concept study. Am J Physiol Lung Cell Mol Physiol 2019; 316: L751-L7L6.

19 Schleich FN, Dallinga JW, Henket M, et al. Volatile organic compounds discriminate between eosinophilic and neutrophilic inflammation in vitro. J Breath Res 2016; 10: 016006.

20 Brinkman $\mathrm{P}$, Wagener AH, Hekking PP, et al. Identification and prospective stability of electronic nose (eNose)-derived inflammatory phenotypes in patients with severe asthma. J Allergy Clin Immunol 2019; 143: 1811-1820.

21 Fens N, Roldaan AC, van der Schee MP, et al. External validation of exhaled breath profiling using an electronic nose in the discrimination of asthma with fixed airways obstruction and chronic obstructive pulmonary disease. Clin Exp Allergy 2011; 41: 1371-1378. 
22 Schleich FN, Zanella D, Stefanuto $\mathrm{PH}$, et al. Exhaled volatile organic compounds are able to discriminate between neutrophilic and eosinophilic asthma. Am J Respir Crit Care Med 2019; 200: 444-453.

23 Wilkinson M, Maidstone R, Loudon A, et al. Circadian rhythm of exhaled biomarkers in health and asthma. Eur Respir J 2019; in press [https://doi.org/10.1183/13993003.01068-2019].

24 Members MSIB, Sansone SA, Fan T, et al. The metabolomics standards initiative. Nat Biotechnol 2007; 25: 846-848. 